\title{
Are Multiple Minimal Outgroup Males Readily Associated with Threat?
}

\section{Florian van Leeuwen ${ }^{1, *}$, Tadeg Quillien², Pascal Boyer $^{3}$}

Department of Political Science, Aarhus University, Bartholins Allé 7, DK-8000 Aarhus C, Denmark.

${ }^{2}$ Department of Psychological and Brain Sciences, University of California, Santa Barbara, CA 93106-9660, USA.

${ }^{3}$ Departments of Psychology and Anthropology, Washington University in St. Louis, St. Louis, MO 63130-4899, USA.

"Author for correspondence (f.van.leeuwen.84@gmail.com)

Humans might have a propensity to associate a collective of multiple outgroup males with threat, even in the context of minimally defined groups. We tested this hypothesis using a fear-conditioning paradigm (Study 1) and a signal detection paradigm (Study 2). Results of Study 1 suggest that stimuli showing ingroup males are more easily associated with threat than stimuli showing outgroup males. Results of Study 2 suggest a bias to perceive single males (both ingroup and outgroup) as more threatening than multiple outgroup males. We discuss the contrast with results of previous studies.

\section{Keywords}

perception, threat, minimal groups, error management

\section{Introduction}

There is a substantial amount of psychological research suggesting that outgroup males are often perceived as threatening (Fessler \& Holbrook, 2013; Maner et al., 2005; Miller, Zielaskowski, \& Plant, 2012; Navarrete et al., 2009, 2012; Olsson, Ebert, Banaji, \& Phelps, 2005; Payne, 2001). A collective of multiple outgroup males may be particularly threatening. Multiple individuals who form a coalition tend to be more powerful (due to their numerical advantage) than single individuals and therefore have more ability to inflict harm. Members of species in which social life is highly coalitional should therefore be particularly sensitive to the number of individuals in a coalition.

Consistent with this evolutionary argument, lions and chimpanzees typically restrain from aggression if they do not outnumber the opposite group (McComb, Packer, \& Pusey, 1994; Wilson, Hauser, \& Wrangham, 2001), and human infants as young as 6-12 months-old are able to infer social dominance from relative numerical size (Pun, Birch, \& Baron, 2016). From the perspective that it is functional for threat-detection mechanisms to minimize false negatives (Haselton \& Nettle, 2006), individuals should be more prone to infer the presence of a threat when they perceive collectives of allied individuals than when they perceive single individuals. The current research tests whether humans are prone to perceiving collectives of multiple outgroup males as particularly threatening.

We tested the hypothesis that humans have a tendency to associate multiple outgroup males with threat using a fear-conditioning paradigm (Study 1) and a signal detection paradigm (Study 2). Fear-conditioning paradigms have been used to investigate preparedness for learning to fear particular kinds of stimuli. Signal detection paradigms have been used to investigate tendencies to perceive particular kinds of stimuli as threatening. As previous research suggest that outgroup males may be associated with threat even when the ingroup-outgroup distinction is minimal (Miller et al., 2012; Navarrete et al., 2012), both studies tested the hypothesis using a minimal group manipulation (Tajfel, Billig, Bundy, \& Flament, 1971).

\section{Study 1}

We first conducted a Pilot Study to verify our ability to observe fear conditioning (see Supplementary materials S1). Study 1 used a similar paradigm to assess fear learning for stimuli showing individual ingroup males, individual outgroup males, multiple ingroup males, and multiple outgroup males.

\section{Method}

We recruited 71 male participants at universities in Lyon, France. Participants were compensated (12 EUR) for their participation. We applied the same exclusion criteria as Olsson et al. (2005), giving $N=66$ (age $M=23.35, S D=$ 7.77).

Before the fear conditioning part of the study, participants were randomly assigned to a green group or an orange group based on a minimal group manipulation (Tajfel et al., 1971). Participants were presented with three pairs of modern art paintings and indicated on an answer sheet for each pair the painting they preferred. The experimenter then ostensibly assessed their scores and then told them that based on their preferences, they were placed in the green (or orange, randomly) group and were asked to put on a green (or orange) sweater.

In the fear-learning paradigm, we used eight pictures showing one or four male-like avatars with neutral facial expressions. The avatars had shirts of the ingroup or outgroup color. The fear conditioning paradigm consisted of three phases. During the habituation phase pictures were presented sequentially on the computer screen, without any sound. There were three habituation trials per picture. During the acquisition phase, for each of the four picture categories, one picture (the conditioned stimulus or $\mathrm{CS}+$ ) was always followed by a $200 \mathrm{~ms}$ loud burst of white noise (the unconditioned stimulus, US) directly after the picture 
presentation, while the other picture of that category was never followed by the US and thus constituted the CS-. (The intensity of the white noise was calibrated by each participant in a work-up procedure before the experiment to be uncomfortable, but not painful.) There were five acquisition trials per picture. The extinction phase was identical to the habituation phase, except that there were six extinction trials per picture. For more information about the method see Supplementary materials S2.

\section{Results}

\section{Ratings of stimuli}

To verify that participants had perceived the stimuli as intended, we analyzed their ratings of the pictures. Participants perceived outgroup avatars as more different than ingroup avatars and perceived multiple avatars as more groupish than single avatars. Furthermore, multiple outgroup avatars were perceived as more dangerous than single outgroup avatars, whereas multiple and single ingroup avatars were perceived equally dangerous (see S2 for analysis).

\section{Skin conductance}

We computed conditioned responses (CRs) as in Olsson et al. (2005). Table 1 shows mean CRs for the habituation, acquisition, and extinction phases, for all four conditions. We observed no significant CRs in the habituation and extinction phases. In the acquisition phase we observed a $\mathrm{CR}$ for pictures of multiple ingroup males and single ingroup males. A two-way repeated-measures ANOVA of the CRs in the acquisition phase revealed no Color $\times$ Number interaction effect, $F(1,65)=0.10, p=.75$, no main effect of number, $F(1,65)<0.01, p=.99$, and a trend for the effect of color, $F(1,65)=2.70, p=.105$.

\section{Study 2}

Study 2 tested the hypothesis using a signal detection paradigm. For social perception, a bias for false alarms might result in perceiving the emotion of anger in actually expressionless faces of outgroup targets associated with threat (Maner et al., 2005).

However, adjusting the perception of anger towards false alarms is not necessarily the optimal design for a threat-detection mechanism. It might be better to bias behavior, rather than perception or encoded information, because this allows the accurate encoded information to be used in other contexts (Perilloux \& Kurzban, 2015). Therefore, it is an empirical question whether a particular error-management strategy involves biased perceptions.

\section{Method}

We recruited 298 participants via Amazon Mechanical Turk. Participants were compensated (US \$0.85) for their participation. We analyzed data for $N=257$ (152 females, 105 males, age $M=35.67, S D=11.60)$. Most participants were White $(n=205)$ or Black/African American $(n=15)$.

Participants were randomly placed in either a Green or Orange group and then completed a signal detection task.

The signal detection task consisted of one practice block of 12 trials and two test blocks of 80 trials. We used 20 pictures showing one or more male-like avatars as background stimuli. All background avatars had a neutral facial expression. We used 20 pictures of human male faces as target stimuli. These target pictures showed 10 males with either neutral or angry expressions.

Background stimuli and targets were varied according to a 2 (target face emotion: neutral vs. anger) $\times 2$ (avatar shirt color: ingroup vs. outgroup) $\times 2$ (avatar number: individual vs. group) within-subjects design. The research question is whether participants have different tendencies to perceive a signal (i.e., perceive an angry face) when the target is accompanied by different background stimuli.

In each test block, there were 10 trials for each cell in the design (in random order). Trials with an angry target stimulus were considered signal trials. The task for participants was to indicate whether the target picture showed an angry facial expression. For details on the method see Supplementary materials S3.

Table 1. Mean conditioned responses (CRs) for the habituation, acquisition, and extinction phases, for all four conditions.

\begin{tabular}{llllll}
\hline Condition & CR & & & \\
Color / Number & $M(S D)$ & $95 \%$ CI for $M$ & $t(65)$ & $p$ & Cohen's $d$ \\
\hline Habituation & & & & & \\
Outgroup / Group & $0.05(0.48)$ & $-0.07,0.16$ & 0.79 & .433 & 0.10 \\
Ingroup / Group & $0.004(0.45)$ & $-0.11,0.16$ & 0.08 & .937 & 0.01 \\
Outgroup / Individual & $0.13(0.52)$ & $-0.00,0.25$ & 1.95 & .056 & 0.24 \\
Ingroup / Individual & $0.01(0.50)$ & $-0.12,0.13$ & 0.08 & .934 & 0.01 \\
\hline Acquisition & & & & & \\
Outgroup / Group & $0.05(0.59)$ & $-0.09,0.20$ & 0.72 & .474 & 0.09 \\
Ingroup / Group & $0.18(0.45)$ & $0.07,0.29$ & 3.22 & .002 & 0.40 \\
Outgroup / Individual & $0.08(0.57)$ & $-0.06,0.21$ & 1.07 & .287 & 0.13 \\
Ingroup / Individual & $0.15(0.46)$ & $0.04,0.27$ & 2.72 & .008 & 0.34 \\
\hline Extinction & & & & & \\
Outgroup / Group & $-0.001(0.46)$ & $-0.11,0.11$ & -0.02 & .981 & -0.003 \\
Ingroup / Group & $-0.05(0.46)$ & $-0.16,0.07$ & -0.83 & .409 & -0.10 \\
Outgroup / Individual & $-0.01(0.46)$ & $-0.12,0.11$ & -0.10 & .917 & -0.01 \\
Ingroup / Individual & $0.02(0.41)$ & $-0.08,0.12$ & 0.40 & .687 & 0.05 \\
\hline
\end{tabular}




\section{Results}

We computed the response bias $c$ using the standard correction for hit rates of 1 and false alarm rates of 0 (Stanislaw \& Todorov, 1999). For the current task, a more negative value of response bias $c$ means that a participant had a stronger tendency to indicate the presence of an angry face (i.e., a lenient threshold for detecting a signal). Thus, for background pictures with multiple outgroup males we predicted lower values for $c$.

Response bias $c$ scores were analyzed with a 2 (color: ingroup vs. outgroup; within-subjects) $\times 2$ (number: individual vs. group; within-subjects) $\times 2$ (participant sex; between-subjects) mixed-design ANOVA that included all interaction effects. The model revealed a significant main effect for number, $F(1,255)=11.825, p=.001$, partial $\eta^{2}$ $=.044$, which was qualified by a 3-way interaction of Sex $\times$ Color $\times$ Number, $F(1,255)=5.757, p=.017$, partial $\eta^{2}=$ .022 . All other $p s>.148$.

We examined estimated marginal means to interpret these effects. The main effect of number indicated that on average, values for $c$ were lower for individual primes $(M$ $=0.294, S E=0.016)$ than for group primes $(M=0.344$, $S E=0.016), p=.001$. The effect of Sex $\times$ Color $\times$ Number indicated that the effect of number differed across the conditions of color and sex (see Figure 1). For female participants, number had a significant effect for outgroup primes $(p<.001)$, but not for ingroup primes $(p=.180)$. In contrast, for male participants, number had no effect for outgroup primes $(p=.954)$ but had a significant effect for ingroup primes $(p=.021)$.

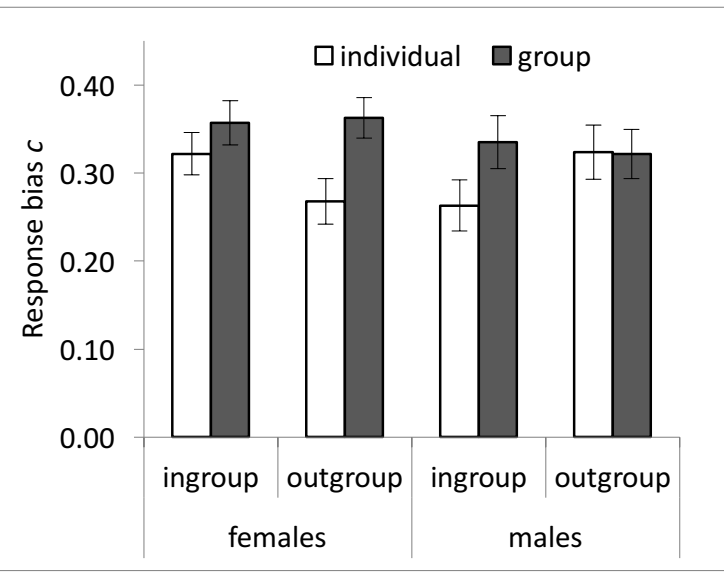

Figure 1. Study 2: Response bias $c$ for female and male participants, for target stimuli following ingroup and outgroup primes displaying individuals and groups. Lower values of $\mathrm{c}$ indicate a stronger tendency to detect an angry face. Error bars show standard errors.

\section{Discussion}

The results of Study 1 provide no support for the hypothesized increased fear learning for stimuli showing multiple outgroup males. We failed to conceptually replicate that in a minimal group context, a single outgroup member is more associated with threat than a single ingroup member. These results contrast with previous findings of increased fear learning (i.e., acquisition) for minimal outgroup targets (Navarrete et al., 2012). The results are broadly in line with recent findings that fear learning for different kinds of outgroups may show learning effects of different strength (Golkar, Björnstjerna, \& Olsson, 2015).

Two differences between the stimuli used in the current study and those by Navarrete et al. (2012) point to an explanation for the inconsistent findings. First, in our study the US consisted only of a burst of noise, whereas Navarrete et al. used both noise and an electric shock. Second, Navarrete et al. used pictures that showed one male each, with only a part of their torso. A classic finding on avoidance learning is that not all CS are equally easily associated with a US (i.e., rats more easily learn to associate taste with nausea than they learn to associate bright-light-plus-noise with nausea; Garcia \& Koelling, 1966). Similarly, minimal outgroup males might be more easily associated with an aversive stimulus when the males are perceived as nearby and the aversive stimulus is tactile.

The results of Study 2 did not support the hypothesized bias to perceive a threat when presented with pictures showing multiple outgroup males. In contrast to Study 1 , the results did not show an effect of color (i.e., values for $c$ did not differ for ingroup and outgroup targets). However, as Study 2 did not contain a manipulation check for explicit identification with the ingroup and outgroup avatars, the absence of an effect for the minimal group manipulation should be interpreted tentatively. Nevertheless, the main effect observed in Study 2 was for the number manipulation and was opposite to our prediction: participants were less likely to perceive threats after seeing images with multiple males.

Two differences in methodology might explain the inconsistent findings. Studies that have observed increased threat perceptions for outgroup males have typically used stimuli referring to existing racial categories (e.g., Correll, Wittenbrink, Park, Judd, \& Goyle, 2011; Maner et al., 2005 ) and/or involved a weapon-detection task (Miller et al., 2012). In contrast, the current study involved minimal groups and a task of detecting facial expressions. Although previous research suggests that threat detection processes may bias the perception of emotion in faces, it might be that such processes of over-perceiving emotion in faces do not generalize to signal detection tasks involving minimal groups. Furthermore, there is ongoing debate about whether perception is influenced at all by top-down cognitive and motivational processes (Firestone \& Scholl, 2016).

In summary, we failed to find evidence that minimal cues of outgroup membership increase fear learning and threat perception. Additionally, we failed to find evidence that another theoretically relevant variable, the numerical size of an outgroup, increases fear learning and threat perception. This suggests that previous findings of biased fear learning and threat perception for minimal outgroup targets are not easy to replicate conceptually, i.e., with comparable but slightly different experimental methods.

\section{Acknowledgement}

The research was supported by a "Package d'Accueil" grant from Université de Lyon (Programme d'Avenir Lyon Saint-Etienne) to P.B. 
Electronic supplementary materials are available online in the journal's webpage. Data files are also available from the Open Science Framework via https://doi.org/10.17605/ OSF.IO/6A7M4

\section{References}

Correll, J., Wittenbrink, B., Park, B., Judd, C. M., \& Goyle, A. (2011). Dangerous enough: moderating racial bias with contextual threat cues. Journal of Experimental Social Psychology, 47, 184-189. (doi: 10.1016/j.jesp.2010.08.017)

Fessler, D. M. T., \& Holbrook, C. (2013) Friends shrink foes: the presence of comrades decreases the envisioned physical formidability of an opponent. Psychological Science, 24, 797-802. (doi: 10.1177/0956797612461508)

Firestone, C., \& Scholl, B. J. (2016). Cognition does not affect perception: evaluating the evidence for "topdown" effects. Behavioral and Brain Sciences, 39, e229. (doi: 10.1017/S0140525X15000965)

Garcia, J., \& Koelling, R. A. (1966). Relation of cue to consequence in avoidance learning. Psychonomic Science, 4, 123-124. (doi: 10.3758/BF03342209)

Golkar, A., Björnstierna, M., \& Olsson, A. (2015). Learned fear to social out-group members are determined by ethnicity and prior exposure. Frontiers in Psychology, 6, 123. (doi: 10.3389/fpsyg.2015.00123)

Haselton, M. G., \& Nettle, D. (2006). The paranoid optimist: an integrative evolutionary model of cognitive biases. Personality and Social Psychology Review, 10, 47-66. (doi: 10.1207/ s15327957pspr1001_3)

Maner, J. K., Kenrick, D. T., Becker, D. V., Robertson, T. E., Hofer, B., Neuberg, S. L., . . Schaller, M. (2005). Functional projection: how fundamental social motives can bias interpersonal perception. Journal of Personality and Social Psychology, 88, 63-78. (doi: 10.1037/0022-3514.88.1.63)

McComb, K., Packer, C., \& Pusey, A. (1994). Roaring and numerical assessment in contests between groups of female lions, Panthera leo. Animal Behaviour, 47, 379387. (doi: 10.1006/anbe.1994.1052)

Miller, S. L., Zielaskowski, K., \& Plant, E. A. (2012). The basis of shooter biases: beyond cultural stereotypes. Personality and Social Psychology Bulletin, 38, 13581366. (doi: 10.1177/0146167212450516)

Navarrete, C. D., McDonald, M. M., Asher, B. D., Kerr, N. L., Yokota, K., Olsson, A., \& Sidanius, J. (2012). Fear is readily associated with an outgroup face in a minimal group context. Evolution and Human Behavior, 33, 590-593. (doi: 10.1016/ j.evolhumbehav.2012.02.007)

Navarrete, C. D., Olsson, A., Ho, A. K., Mendes, W. B., Thomsen, L., \& Sidanius, J. (2009). Fear extinction to an out-group face: the role of target gender. Psychological Science, 20, 155-158. (doi: 10.1111/ j.1467-9280.2009.02273.x)

Olsson, A., Ebert, J. P., Banaji, M. R., \& Phelps, E. A. (2005). The role of social groups in the persistence of learned fear. Science, 309, 785-787. (doi: 10.1126/ science.1113551)

Payne, B. K. (2001). Prejudice and perception: the role of automatic and controlled processes in misperceiving a weapon. Journal of Personality and Social Psychology, 81, 181-192. (doi: 10.1037/0022-3514.81.2.181)

Perilloux, C., \& Kurzban, R. (2015). Do men overperceive women's sexual interest? Psychological Science, 26, 70-77. (doi: 10.1177/0956797614555727)

Pun, A., Birch, S. A. J., \& Baron, A. S. (2016). Infants use relative numerical group size to infer social dominance. Proceedings of the National Academy of Sciences of the United States of America, 113, 23762381. (doi: 10.1073/pnas.1514879113)

Stanislaw, H., \& Todorov, N. (1999). Calculation of signal detection theory measures. Behavior Research Methods, Instruments, \& Computers, 31, 137-149. (doi: 10.3758/BF03207704)

Tajfel, H., Billig, M. G., Bundy, R. P., \& Flament, C. (1971). Social categorization and intergroup behaviour. European Journal of Social Psychology, 1, 149-178. (doi: 10.1002/ejsp.2420010202)

Wilson, M. L., Hauser, M. D., \& Wrangham, R. W. (2001). Does participation in intergroup conflict depend on numerical assessment, range location, or rank for wild chimpanzees? Animal Behaviour, 61, 1203-1216. (doi: 10.1006/anbe.2000.1706) 